\title{
Insufficient evidence on diagnostic accuracy of radiographic methods in periapical tissues
}

\author{
Abstracted from \\ Petersson A, Axelsson S, Davidson T, et al. \\ Radiological diagnosis of periapical bone tissue lesions in endodontics: \\ a systematic review. Int Endod / 2012; 45: 783-801. \\ Address for correspondence: Arne Petersson, Department of Oral and Maxillofacial Radiology, \\ Faculty of Odontology, Malmo“" University, SE-205 06 Malmo“, Sweden. E-mail: arne.petersson@mah.se
}

\section{Question: What is the diagnostic accuracy of available radiographic methods in use for imaging the periapical bone tissue area?}

Data sources The Cochrane Central register of controlled trials, Medline and Embase databases were searched.

Study selection Clinical studies that compared methods against a reference standard were included. Studies also needed to have sensitivity, specificity, likelihood ratio, odds ratio or receiver operator curve (ROC) data or data reported so this could be calculated.

Data extraction and synthesis Two reviewers independently assessed abstracts and full text articles. Study quality was assessed using the GRADE criteria (www.gradeworkinggroup.org/). A qualitative summary was conducted.

Results Twenty-six studies were included, none was considered to be of high quality, 11 were of moderate quality. There was insufficient evidence that the digital intraoral radiographic technique is diagnostically as accurate as the conventional film technique. The same applies to Cone beam computed tomography (CBCT).

Conclusions No conclusions can be drawn regarding the accuracy of radiological examination in identifying various forms of periapical bone tissue changes or about the pulpal condition.

\section{Commentary}

Tooth preservation is an important goal for endodontic therapy. The use of radiographs (conventional or digital) is an adjunctive tool for diagnosis when apical pathology is suspected. However, the magnitude and the nature of the pathology cannot be determined by only using a 2D image. New techniques have emerged, such $\mathrm{CBCT}$, that claim 3D images are more beneficial for these types of diagnosis.

The systematic review intended to evaluate the accuracy of different radiographic methods and their ability to evaluate presence, absence and changes over time of a periapical lesion. In addition, it looks into what type of information may be garnered from the radiographic images as to the evolution and condition of the pulp in teeth without endodontic treatment.
They have four different questions to answer related to the same topic. A reasonable number of databases were searched, however only abstracts in English were accepted. A flowchart followed to assess the progress and the final selection of the articles for the review. The information of the selected article was presented in a very explicit table and an evidence grading system (QUADAS) was used to assess the quality of the individual studies. Results were only expressed in terms of each individual study. Narrative assessment results were given for each individual hypothesis for the four questions presented originally. Some statistics are presented, however it is unclear how they were obtained. It is noted this review is part of a bigger publication 'Methods of Diagnosis and Treatment in Endodontics' performed by the Swedish Council on Health Technology Assessment (http://www.sbu.se/upload/Publikationer/ Content1/1/endodontics_eng_smf.pdf) The relevant issue is that the review included human clinical trials with ex-vivo (postmortem) trials. As important as they are, post-mortem trials and their results cannot be compared and applied directly in clinical practice.

It is important for us, as clinicians making daily decisions, to know the source of results in the literature. Purchasing and incorporating new radiographic technology and instituting techniques in our practices is very important, only if they can improve diagnosis accuracy. If this is possible it will avoid pain and suffering and halt the progression of unwanted diseases. However, the use of radiation for the purpose of diagnosis is not a free ride such as the use of СBCT for smaller lesions; the benefits have to outweigh the risks in order to justify the use of it.

\section{Practice point}

- With the onset of new radiographic technology such as digital and CT scan, studies should be geared towards evaluating these methods compared to traditional radiographs. It would be invaluable to a clinician to be able to predict the outcome/ success of endodontic treatment based on what is visualised on the radiograph.

Debra M Ferraiolo

NYU College of Dentistry, New York, USA

Evidence-Based Dentistry (2012) 13, 106. doi:10.1038/sj.ebd.6400890 